\title{
Produtividade de pepino para processamento no Vale do São Francisco(1)
}

\author{
Geraldo Milanez de Resende(2) e José Egidio Flori(2)
}

\begin{abstract}
Resumo - Com o objetivo de identificar as cultivares mais produtivas de pepino para processamento, instalaram-se dois experimentos de maio a agosto de 1997, em Petrolina, PE. O delineamento experimental utilizado foi em blocos ao acaso, com 18 tratamentos e três repetições. A parcela foi composta de quatro linhas de 3,0 m de comprimento, com espaçamento de 1,0x0,30 m. Os frutos comerciais foram classificados na categoria conserva com 6 a $12 \mathrm{~cm}$, e categoria cornichon, com 4,0 a 5,5 cm de comprimento. Na categoria conserva, as cultivares Eureka (34,54 t/ha) e Francipak (33,72 t/ha) destacaram-se em termos de produtividade. As cultivares Pioneiro, Vlaspik e Wisconsin SMR 18 apresentaram os menores desempenhos (21,43 a 23,45 t/ha). Verificou-se uma variação de 30,74 a 37,79 g/fruto e 9,01 a 14,81 frutos/planta. Na categoria cornichon, destacaram-se as cultivares Vlasset e Supremo, seguidas de Vlasstar e Prêmio, com produtividades acima de 10,0 t/ha. O pior desempenho foi da cultivar Wisconsin SMR 18, cuja produção de frutos foi 5,61 t/ha. Foram obtidas variações de 6,09 a 7,27 g/fruto e 11,48 a 24,15 frutos/planta.
\end{abstract}

Termos para indexação: Cucumis sativus, hortaliça, rendimento.

\section{Productivity of pickling cucumber in the São Francisco Valley, Brazil}

\begin{abstract}
With the objective of indicating pickling cucumber cultivars of high productivity, two field experiments were carried out in Petrolina, Pernambuco State, Brazil, from May to August of 1997. The experimental design was a randomized complete block with 18 treatments and three replications. Experimental plots consisted of four rows of $3 \mathrm{~m}$ long, and the plants were on a $1.00 \times 0.30 \mathrm{~m}$ spacing grid. The commercial fruits were classified as pickling (fruits length from 6 to $12 \mathrm{~cm}$ ) and cornichon (from 4.0 to $5.5 \mathrm{~cm})$. The cultivars Eureka $(34.54 \mathrm{t} / \mathrm{ha})$ and Francipak $(33.72 \mathrm{t} / \mathrm{ha})$ presented the highest yield for pickling. The cultivars Pioneiro, Vlaspik and Wisconsin SMR 18 showed the lowest yield varying from 21.43 to 23.45 t/ha. There was a variation from 30.74 to $37.79 \mathrm{~g} /$ fruit and from 9.01 to 14.81 fruit/plant. For cornichon stood out the cultivars Vlasset and Supremo, followed by Vlasstar and Prêmio which showed yield above 10.00 t/ha. The lowest yield was presented by Wisconsin SMR 18, with $5.61 \mathrm{t} / \mathrm{ha}$. There was a variation from 6.09 to $7.27 \mathrm{~g} /$ fruit and 11.48 to 24.15 fruits/plant.
\end{abstract}

Index terms: Cucumis sativus, vegetables, yield.

\section{Introdução}

A cultura do pepino para processamento ocupa posição de destaque no Sul do Brasil, sendo o Estado de Santa Catarina o principal produtor nacional, com produtividade média de 10,0 t/ha (Silva et al., 1992). Em nível internacional, Cerne (1994), trabalhando com a cultivar Levina, obteve produtividade de 31,4 t/ha de frutos comerciais e Cerne et al.

\footnotetext{
(1) Aceito para publicação em 27 de setembro de 2002.

${ }^{(2)}$ Embrapa-Centro de Pesquisa Agropecuária do Trópico SemiÁrido, Caixa Postal 23, CEP 56300-970 Petrolina, PE. E-mail gmilanez@cpatsa.embrapa.br, jeflori@cpatsa.embrapa.br
}

(2000), avaliando diferentes cultivares, obtiveram produtividade média de $40,7 \mathrm{t} / \mathrm{ha}$, destacando-se a cultivar Harmonie como a mais produtiva, com 45,4 t/ha.

Alguns autores têm estudado a cultura do pepino para conserva, cuja principal característica é a maior produtividade de frutos de tamanho pequeno (4 a $12 \mathrm{~cm}$ ), considerados ideais para processamento industrial. Silva et al. $(1988,1992)$ encontraram valores médios com as cultivares Score e Ginga AG-77, de 23,8 t/ha de frutos com 6 a $12 \mathrm{~cm}$ de comprimento, com $357 \mathrm{~g} /$ planta, massa fresca do fruto de $33,0 \mathrm{~g}$ e 10,8 frutos/planta, mas não relataram quanto aos valores dessas características por cultivar. Resende 
(1999) encontrou produtividades de 13,9 (cultivar Pérola) a 29,7 t/ha (cultivar Indaial), destacando também as cultivares Score (26,5 t/ha), Colônia (26,4 t/ha) e Ginga AG-77 (26,1 t/ha); a massa fresca do fruto variou de 49,2 a 67,4 g/fruto (cultivares Primepak e Pérola, respectivamente) e o número de frutos por planta foi de 3,2 a 7,7 (cultivares Pérola e Indaial, respectivamente). North Caroline Cooperative Extension Service (2000) recomendou para cultivo de pepino para conserva as cultivares Calypso, Francipak, Flurry, Navigator, Vlaspik, Vlasset e Vlasstar.

Resende \& Pessoa (1996), utilizando a classificação cornichon, com frutos comerciais variando de 4,0 a $5,5 \mathrm{~cm}$ de comprimento, destacaram as cultivares Tamor (10,2 t/ha), Indaial (10,1 t/ha), Colônia (10,1 t/ha), Score (9,8 t/ha), Ginga AG-77 (9,7 t/ha), Levina (9,6 t/ha) e Primepak (8,8 t/ha) como as mais produtivas. A massa fresca do fruto variou de 5,6 a 6,1 g/fruto (cultivares Score e Primepak) e o número de frutos por plantas variou de 8,3 a 27,8 frutos/ planta (cultivares Pérola e Tamor, respectivamente). A porcentagem de frutos não comerciais variou de $11,4 \%$ a $16,1 \%$ (cultivares Pérola e Levina, respectivamente).

No Nordeste do Brasil há grande deficiência de oleráceas para cultivo em áreas irrigadas que possam ser processadas industrialmente e utilizar a infraestrutura agroindustrial já instalada na região. De acordo com Rabelo et al. (1990), existe grande deficiência na produção e qualidade das matériasprimas nos pólos de irrigação, onde um pequeno número de culturas industriais (tomate e aspargo) obriga as agroindústrias a operarem com ociosidade ou a importarem matéria-prima de outras regiões. Assim, a identificação de novas opções de oleráceas que possam ser processadas industrialmente é uma das prioridades para as áreas irrigadas do Nordeste.

Este trabalho objetivou identificar cultivares de pepino mais produtivas para processamento e com maior quantidade de frutos tipo conserva e cornichon nas condições do Vale do São Francisco, como alternativa agroindustrial para a região.

\section{Material e Métodos}

Dois experimentos foram realizados no Campo Experimental de Bebedouro, Embrapa-Centro de Pesquisa Agropecuária do Trópico Semi-Árido, Petrolina, PE, lati- tude $9^{\circ} 9^{\prime} \mathrm{S}$, longitude $40^{\circ} 29^{\prime} \mathrm{O}$ e altitude de $365,5 \mathrm{~m}$ (Amorim Neto, 1989), em Latossolo Vermelho-Amarelo, com as seguintes características $(0-20 \mathrm{~cm}): \mathrm{pH}\left(\mathrm{H}_{2} \mathrm{O}\right), 6,5$; $\mathrm{Ca}^{2+}, 2,3 \mathrm{cmol}_{\mathrm{c}} / \mathrm{dm}^{3} ; \mathrm{Mg}^{2+}, 0,8 \mathrm{cmol}_{\mathrm{c}} / \mathrm{dm}^{3} ; \mathrm{Na}^{+}$, $0,04 \mathrm{cmol}_{\mathrm{C}} / \mathrm{dm}^{3} ; \mathrm{K}^{+}, 0,32 \mathrm{cmol}_{\mathrm{C}} / \mathrm{dm}^{3} ; \mathrm{Al}^{3}, 0,04 \mathrm{cmol}_{\mathrm{C}} / \mathrm{dm}^{3}$, e P (Mehlich), 23,78 mg/L, determinadas segundo Embrapa (1979).

O delineamento experimental utilizado nos dois experimentos foi de blocos ao acaso, com 18 cultivares (Calypso, Eureka, Flurry, Francipak, Ginga AG-77, Imperial, Nautillus, Navigator, Panorama, Pioneiro, Premier, Prêmio, Primepak, Wisconsin SMR 18, Supremo, Vlaspik, Vlasset e Vlasstar) e três repetições. À exceção da cultivar Wisconsin SMR 18, de polinização aberta, todas as demais eram híbridos. As parcelas experimentais constaram de quatro linhas de $3,0 \mathrm{~m}$, com espaçamento de $1,0 \times 0,30 \mathrm{~m}$, com duas plantas por cova, as linhas centrais constituindo a área útil e as linhas laterais a bordadura, e a semeadura feita em 25 de maio de 1997.

Nas adubações de plantio, feitas com base na análise de solo, utilizaram-se $150 \mathrm{~kg} / \mathrm{ha}$ de sulfato de amônio, $220 \mathrm{~kg} / \mathrm{ha}$ de superfosfato simples e $65 \mathrm{~kg} / \mathrm{ha}$ de cloreto de potássio. Também foram realizadas duas adubações em cobertura com $150 \mathrm{~kg} / \mathrm{ha}$ de sulfato de amônio, a primeira aos 21 dias da semeadura, após o desbaste, e a segunda 15 dias depois.

Foram realizadas irrigações por aspersão três vezes por semana, e manteve-se a cultura limpa mediante capinas manuais e conduzida sob sistema rasteiro. Os tratos fitossanitários foram os comumente recomendados para a cultura do pepino, realizados somente até o início da floração (34 dias após a semeadura), em razão dos curtos períodos das colheitas. Após o início da floração, utilizaram-se somente produtos à base de enxofre e detergente neutro, pulverizados semanalmente até o fim da colheita.

As colheitas foram feitas diariamente para a classificação cornichon e três vezes por semana para a classificação conserva, iniciando-se aos 40 e 43 dias, respectivamente, após a semeadura, e estendendo-se por 38 dias. Foram avaliados a produtividade comercial ( $\mathrm{t} / \mathrm{ha}$ ), massa fresca de fruto $(\mathrm{g})$ e número de frutos por planta, e realizada a classificação de frutos comerciais em cornichon e conserva. Frutos não deformados, retilíneos, sem manchas e sem lesões foram considerados comerciais e classificados. A categoria conserva foi dividida em classe 1: frutos com 6 a $9 \mathrm{~cm}$, e classe 2: frutos com 9 a $12 \mathrm{~cm}$ de comprimento (Resende \& Pessoa, 1996). A categoria cornichon foi dividida em frutos da classe 1: 4,0 a 4,5 $\mathrm{cm}$, classe $2: 4,5$ a $5,0 \mathrm{~cm}$ e classe 3: 5,0 a 5,5 cm de comprimento (Resende, 1999). Os dados foram submetidos à análise de variância e as médias comparadas pelo teste de Scott-Knott, a 5\% 
de probabilidade. Os dados originais de porcentagem foram transformados em arco seno $(\mathrm{P} / 100)^{0,5}$ para a análise estatística.

\section{Resultados e Discussão}

As maiores produtividades de frutos comerciais para a categoria conserva foram apresentadas pelas cultivares Eureka (34,54 t/ha) e Francipak (33,72 t/ha), que não superaram a produtividade média de 40,66 t/ha relatada por Cerne et al. (2000) (Tabela 1). As cultivares Vlasset, Calypso, Flurry, Primepak, Ginga AG-77, Panorama e Supremo, com produtividades acima de 28,0 t/ha, são similares às relatadas por Resende (1999), que obteve até $29,72 \mathrm{t} / \mathrm{ha}$ com a cultivar Indaial. A cultivar Wisconsin SMR 18, Pioneiro e Vlaspik foram as menos produtivas, com valores inferiores à produtividade média das cultivares Score e Ginga AG-77, de 23,8 t/ha, obtidas por Silva et al. (1992).

A maior massa fresca de frutos foi apresentada pela cultivar Francipak (37,79 g/fruto), não diferin- do estatisticamente das cultivares Flurry, Vlaspik, Vlasstar, Eureka, Ginga AG-77, Supremo e Navigator (34,05 a 36,24 g/fruto), mas inferior às estudadas em Minas Gerais por Resende (1999), que obteve valores entre 49,17 g/fruto com 'Primepak' a 67,35 g/fruto com 'Pérola'.

A cultivar Eureka apresentou maior número de frutos por planta $(14,81)$, sem diferir estatisticamente das cultivares Calypso, Primepak, Vlasset, Francipak, Imperial, Ginga AG-77, Flurry e Panorama. Esses resultados são superiores aos 10,8 frutos/planta obtidos por Silva et al. (1992), com as cultivares Score e Ginga AG-77, e aos de Cantliffe \& Phatak (1975), 6,3 frutos/planta com a cultivar Premier. A cultivar Wisconsin SMR 18 (9,90 frutos/ planta), Pioneiro (9,37 frutos/planta) e Vlaspik (9,01 frutos/planta) apresentaram o menor número de frutos, mas ainda assim superiores aos obtidos por Resende (1999), que registrou variações de 3,18 com 'Pérola' a 7,72 frutos/planta com 'Indaial'.

Em relação à classificação dos frutos, verificouse maior proporção de frutos classe 1 , de maior cota-

Tabela 1. Produtividade comercial, massa fresca dos frutos, número de frutos por planta e classificação de frutos comerciais de cultivares de pepino para processamento, categoria conserva. Petrolina, PE, 1997(1).

\begin{tabular}{|c|c|c|c|c|c|}
\hline Cultivar & $\begin{array}{c}\text { Produtividade } \\
\text { comercial (t/ha) }\end{array}$ & $\begin{array}{l}\text { Massa fresca } \\
\text { dos frutos }(\mathrm{g})\end{array}$ & $\begin{array}{l}\text { Número de } \\
\text { frutos/planta }\end{array}$ & $\begin{array}{c}\text { Classificação de frutos } \\
\text { Classe } 1 \\
\end{array}$ & $\begin{array}{c}\text { comerciais }(\%)^{(2)} \\
\text { Classe } 2 \\
\end{array}$ \\
\hline Eureka & $34,54 \mathrm{a}$ & $34,93 \mathrm{a}$ & $14,81 \mathrm{a}$ & $85,66 c$ & $14,34 \mathrm{a}$ \\
\hline Francipak & $33,72 \mathrm{a}$ & $37,79 \mathrm{a}$ & $13,50 \mathrm{a}$ & $87,96 \mathrm{c}$ & $12,04 \mathrm{a}$ \\
\hline Vlasset & $31,28 b$ & $33,57 b$ & $13,99 \mathrm{a}$ & $92,21 b$ & $7,79 \mathrm{~b}$ \\
\hline Calypso & $31,08 \mathrm{~b}$ & $33,19 b$ & $14,03 \mathrm{a}$ & $90,66 b$ & $9,34 b$ \\
\hline Flurry & $30,81 b$ & $36,24 a$ & $12,89 \mathrm{a}$ & $87,00 \mathrm{c}$ & $13,00 \mathrm{a}$ \\
\hline Primepak & $29,37 b$ & $32,04 \mathrm{~b}$ & $14,00 \mathrm{a}$ & $90,86 b$ & $9,14 \mathrm{~b}$ \\
\hline Ginga AG-77 & $29,35 b$ & $34,06 \mathrm{a}$ & $12,93 \mathrm{a}$ & $91,87 \mathrm{~b}$ & $8,13 b$ \\
\hline Panorama & $28,37 \mathrm{~b}$ & $33,25 b$ & $12,72 \mathrm{a}$ & $86,89 \mathrm{c}$ & $13,11 \mathrm{a}$ \\
\hline Supremo & $28,33 b$ & $34,06 \mathrm{a}$ & $11,89 \mathrm{~b}$ & $92,20 \mathrm{~b}$ & $7,80 \mathrm{~b}$ \\
\hline Vlasstar & $27,43 \mathrm{c}$ & $35,43 a$ & $11,63 b$ & $88,43 \mathrm{c}$ & $11,57 \mathrm{a}$ \\
\hline Imperial & $26,84 \mathrm{c}$ & $30,22 b$ & $13,22 \mathrm{a}$ & $93,54 a$ & $6,46 \mathrm{c}$ \\
\hline Navigator & $25,32 \mathrm{c}$ & $34,05 \mathrm{a}$ & $11,18 b$ & $91,01 b$ & $8,99 b$ \\
\hline Premier & $25,11 \mathrm{c}$ & $32,19 b$ & $12,00 \mathrm{~b}$ & $92,34 \mathrm{~b}$ & $7,66 b$ \\
\hline Nautillus & $24,77 \mathrm{c}$ & $32,54 \mathrm{~b}$ & $12,07 b$ & $95,27 \mathrm{a}$ & $4,73 \mathrm{c}$ \\
\hline Prêmio & $24,23 \mathrm{c}$ & $32,60 \mathrm{~b}$ & $11,16 \mathrm{~b}$ & $90,24 b$ & $9,76 \mathrm{~b}$ \\
\hline Vlaspik & $23,45 \mathrm{~d}$ & $36,07 \mathrm{a}$ & $9,90 \mathrm{c}$ & $85,83 \mathrm{c}$ & $14,17 \mathrm{a}$ \\
\hline Wisconsin SMR 18 & $21,77 d$ & $30,74 b$ & $9,01 \mathrm{c}$ & $86,37 \mathrm{c}$ & $13,63 \mathrm{a}$ \\
\hline Pioneiro & $21,43 \mathrm{~d}$ & $30,74 \mathrm{~b}$ & $9,37 \mathrm{c}$ & $86,47 \mathrm{c}$ & $13,53 \mathrm{a}$ \\
\hline $\mathrm{CV}(\%)$ & 7,36 & 5,04 & 9,28 & 2,38 & 8,91 \\
\hline
\end{tabular}

${ }^{(1)}$ Médias seguidas de mesma letra, nas colunas, não diferem entre si pelo teste Scott-Knott a $5 \%$ de probabilidade. ${ }^{(2)}$ Os frutos comerciais foram classificados em classe 1 com 6 a $9 \mathrm{~cm}$ e classe 2 com 9 a $12 \mathrm{~cm}$ de comprimento. 
ção no mercado, em todos os materiais (Tabela 1). Embora as cultivares Nautillus $(95,27 \%)$ e Imperial $(93,54 \%)$ tenham se sobressaído em relação às demais, observou-se pequena variação entre os materiais testados, demonstrando padrão de qualidade no que se refere à produção de frutos adequados ao processamento na forma de conserva. Resende (1999) obteve com a melhor cultivar (Ginga AG-77) até $78 \%$ de frutos classe 1 e Silva et al. (1979) máximo de $66,57 \%$ com a cultivar Premier. Em frutos classe 2 verificou-se uma variação de $4,73 \%$ a $14,34 \%$, resultados inferiores aos observados por Resende (1999), que verificou oscilações entre $22,04 \%$ a $33,78 \%$.

$\mathrm{Na}$ categoria cornichon, as cultivares Vlasset, Vlasstar, Prêmio e Supremo, com produtividades acima de 10,0 t/ha, se destacaram, mas não mostra- ram diferenças significativas das cultivares Primepak, Ginga AG-77, Calypso, Eureka, Imperial, Francipak, Nautillus, Premier e Panorama (Tabela 2). Esses resultados são semelhantes aos de Resende \& Pessoa (1996), os quais também verificaram produtividades de até $10,0 \mathrm{t} / \mathrm{ha}$. A cultivar Wisconsin SMR 18 foi a menos produtiva, com $5,61 \mathrm{t} / \mathrm{ha}$

As maiores massas frescas de frutos da categoria cornichon foram observadas nas cultivares Nautillus (7,27 g/fruto), Wisconsin SMR 18 (7,17 g/fruto), Supremo ( $7,11 \mathrm{~g} /$ fruto), Vlasset (6,96 g/fruto) e Navigator $(6,94 \mathrm{~g} /$ fruto), superiores às variações de 5,64 a 6,08 frutos/planta citadas por Resende \& Pessoa (1996).

Em relação ao número de frutos por planta, os maiores valores foram evidenciados pelas cultiva-

Tabela 2. Produtividade comercial, massa fresca dos frutos, número de frutos por planta e classificação de frutos comerciais de cultivares de pepino para processamento, categoria cornichon. Petrolina, PE, $1997^{(1)}$.

\begin{tabular}{lcccccc}
\hline Cultivar & Produtividade & Massa fresca & Número de & \multicolumn{2}{c}{ Classificação de frutos (\%) } \\
\cline { 5 - 6 } & comercial (t/ha) & dos frutos $(\mathrm{g})$ & frutos/planta & Classe 1 & Classe 2 & Classe 3 \\
\hline Vlasset & $10,39 \mathrm{a}$ & $6,96 \mathrm{a}$ & $21,87 \mathrm{a}$ & $67,58 \mathrm{a}$ & $21,64 \mathrm{~b}$ & $10,78 \mathrm{~b}$ \\
Vlasstar & $10,30 \mathrm{a}$ & $6,65 \mathrm{~b}$ & $24,15 \mathrm{a}$ & $61,27 \mathrm{~b}$ & $24,65 \mathrm{a}$ & $14,08 \mathrm{a}$ \\
Prêmio & $10,12 \mathrm{a}$ & $6,72 \mathrm{~b}$ & $22,62 \mathrm{a}$ & $61,34 \mathrm{~b}$ & $22,73 \mathrm{~b}$ & $15,93 \mathrm{a}$ \\
Supremo & $10,05 \mathrm{a}$ & $7,11 \mathrm{a}$ & $23,32 \mathrm{a}$ & $62,88 \mathrm{~b}$ & $22,86 \mathrm{~b}$ & $14,26 \mathrm{a}$ \\
Primepak & $9,98 \mathrm{a}$ & $6,63 \mathrm{~b}$ & $23,43 \mathrm{a}$ & $60,96 \mathrm{~b}$ & $25,81 \mathrm{a}$ & $13,23 \mathrm{~b}$ \\
Ginga AG-77 & $9,93 \mathrm{a}$ & $6,64 \mathrm{~b}$ & $20,62 \mathrm{~b}$ & $64,61 \mathrm{a}$ & $21,20 \mathrm{~b}$ & $14,19 \mathrm{a}$ \\
Calypso & $9,85 \mathrm{a}$ & $6,71 \mathrm{~b}$ & $22,04 \mathrm{a}$ & $60,33 \mathrm{~b}$ & $24,04 \mathrm{a}$ & $15,63 \mathrm{a}$ \\
Eureka & $9,82 \mathrm{a}$ & $6,82 \mathrm{~b}$ & $19,66 \mathrm{~b}$ & $66,02 \mathrm{a}$ & $21,21 \mathrm{~b}$ & $12,77 \mathrm{~b}$ \\
Imperial & $9,73 \mathrm{a}$ & $6,79 \mathrm{~b}$ & $21,52 \mathrm{a}$ & $61,04 \mathrm{~b}$ & $25,55 \mathrm{a}$ & $13,41 \mathrm{~b}$ \\
Francipak & $9,69 \mathrm{a}$ & $6,52 \mathrm{~b}$ & $21,78 \mathrm{a}$ & $62,27 \mathrm{~b}$ & $24,91 \mathrm{a}$ & $12,82 \mathrm{~b}$ \\
Nautillus & $9,65 \mathrm{a}$ & $7,27 \mathrm{a}$ & $19,56 \mathrm{~b}$ & $62,27 \mathrm{~b}$ & $22,96 \mathrm{~b}$ & $14,77 \mathrm{a}$ \\
Premier & $9,47 \mathrm{a}$ & $6,42 \mathrm{~b}$ & $21,74 \mathrm{a}$ & $61,10 \mathrm{~b}$ & $24,37 \mathrm{a}$ & $14,53 \mathrm{a}$ \\
Panorama & $9,08 \mathrm{a}$ & $6,50 \mathrm{~b}$ & $20,53 \mathrm{~b}$ & $68,08 \mathrm{a}$ & $21,17 \mathrm{~b}$ & $10,75 \mathrm{~b}$ \\
Flurry & $8,24 \mathrm{~b}$ & $6,09 \mathrm{~b}$ & $17,49 \mathrm{c}$ & $54,79 \mathrm{~d}$ & $28,18 \mathrm{a}$ & $17,03 \mathrm{a}$ \\
Pioneiro & $8,19 \mathrm{~b}$ & $6,71 \mathrm{~b}$ & $15,96 \mathrm{c}$ & $62,89 \mathrm{~b}$ & $22,15 \mathrm{~b}$ & $14,96 \mathrm{a}$ \\
Vlaspik & $8,13 \mathrm{~b}$ & $6,60 \mathrm{~b}$ & $17,80 \mathrm{c}$ & $62,84 \mathrm{~b}$ & $24,64 \mathrm{a}$ & $12,52 \mathrm{~b}$ \\
Navigator & $7,61 \mathrm{~b}$ & $6,94 \mathrm{a}$ & $16,75 \mathrm{c}$ & $63,46 \mathrm{~b}$ & $23,25 \mathrm{~b}$ & $13,29 \mathrm{~b}$ \\
Wisconsin SMR 18 & $5,61 \mathrm{c}$ & $7,17 \mathrm{a}$ & $11,48 \mathrm{~d}$ & $58,90 \mathrm{c}$ & $23,78 \mathrm{a}$ & $17,32 \mathrm{a}$ \\
\hline CV (\%) & 7,31 & 3,25 & 6,99 & 2,56 & 3,81 & 6,23 \\
\hline
\end{tabular}

${ }^{(1)}$ Médias seguidas de mesma letra, nas colunas, não diferem entre si pelo teste Scott-Knott a $5 \%$ de probabilidade. (2) Os frutos comerciais foram classificados em classe $1 \mathrm{com} \mathrm{4,0} \mathrm{a} \mathrm{4,5} \mathrm{cm;} \mathrm{classe} 2 \mathrm{com} \mathrm{4,5}$ a 5,0 cm e classe $3 \mathrm{com} 5,0$ a 5,5 cm de comprimento. 
res Vlasstar (24,15 frutos/planta), Primepak (23,43 frutos/planta), Supremo (23,32 frutos/planta), Prêmio (22,62 frutos/planta), Calypso (22,04 frutos/planta), Vlasset (21,87 frutos/planta), Francipak (21,78 frutos/planta), Premier (21,74 frutos/planta) e Imperial (21,52 frutos/planta); o pior desempenho foi da cultivar Wisconsin SMR 18 (11,48 frutos/ planta) (Tabela 2). Resultados superiores foram relatados por Resende \& Pessoa (1996), com os maiores valores entre 25,22 com 'Levina' e 27,78 frutos/planta com 'Tamor'.

Na classificação de frutos comerciais, verificouse maior proporção de frutos classe 1, de maior cotação no mercado, em todas as cultivares, alcançando valores superiores a 58\% (Tabela 2). As cultivares Panorama $(68,08 \%)$, Vlasset $(67,58 \%)$, Eureka $(66,02 \%)$ e Ginga AG-77 $(64,61 \%)$ apresentaram as maiores porcentagens. Para frutos classes 2 e 3 foram obtidas variações de $10,75 \%$ a $28,18 \%$. Resende \& Pessoa (1996) também verificaram nas cultivares Ginga AG-77 e Primepak valores superiores a $60 \%$ em frutos com 4,0 a $4,5 \mathrm{~cm}$ de comprimento, e oscilação de $20,81 \%$ a $28,58 \%$ e $11,96 \%$ a $15,31 \%$ nos frutos com 4,5 a $5,0 \mathrm{~cm}$ e 5,0 a $5,5 \mathrm{~cm}$ de comprimento, respectivamente.

Com relação ao ciclo vegetativo, verificou-se variação de 78 a 81 dias nas cultivares avaliadas para a classificação conserva e cornichon, respectivamente, com nenhuma diferença de ciclo dentro de cultivares que chegasse a viabilizar um provável escalonamento da produção. No que se refere à ocorrência de pragas e doenças, apenas observou-se no final da última semana de colheita pequena incidência de mosca-branca (Bemisia sp.), que, por já estar no fim do ciclo vegetativo, não causou danos à cultura.

\section{Conclusões}

1. As cultivares Eureka e Francipak são as mais indicadas para o cultivo de pepino para processamento categoria conserva.

2. As cultivares Vlasset, Supremo, Vlasstar e Prêmio são as mais indicadas para o cultivo de pepino para processamento tipo cornichon.

\section{Referências}

AMORIM NETO, M. da S. Informações meteorológicas dos campos experimentais de Bebedouro e Mandacaru,
Petrolina, PE. Petrolina: Embrapa-CPATSA, 1989. 58 p. (Documentos, 57).

CANTLIFFE, D. J.; PHATAK, S. C. Plant population studies with pickling cucumbers grown for once-over harvest. Journal of the American Society for Horticultural Science, Mount Vernon, v. 100, n. 5, p. 464466, 1975.

CERNE, M. Different agrotextiles for direct covering of pickling cucumbers. Acta Horticulturae, Skierniewice, v. 371, p. 247-252, 1994.

CERNE, M.; SKOF, M.; UGRINOVIÉ, K. Pickling cucumber (Cucumis sativus L.) cultivars grown in two different ecological conditions. Acta Horticulturae, Bari, v. 533, p. 549-555, 2000.

EMBRAPA. Serviço Nacional de Levantamento e Conservação de Solos (Rio de Janeiro, RJ). Manual de métodos de análise de solo. Rio de Janeiro, 1979. 1 v.

NORTH CAROLINE COOPERATIVE EXTENSION SERVICE. Commercial production of pickling $\&$ slicing cucumbers in North Carolina. Disponível em: http:// www.ces.ncsu.edu.html. Acesso em: 27 mar. 2000.

RABELO, J. L. C.; COELHO, J. P.; SANTOS, J. A. N. Estudos sobre a agroindústria no Nordeste: situação atual a perspectiva da produção irrigada. Fortaleza: Banco do Nordeste do Brasil/Escritório Técnico de Estudos Econômicos do Nordeste, 1990. v. 2.

RESENDE, G. M. de. Produção de pepino para conserva na região Norte de Minas Gerais. Horticultura Brasileira, Brasília, v. 17, n. 1, p. 57-60, maio 1999.

RESENDE, G. M. de; PESSOA, H. B. S. V. Produção de pepino para indústria no perímetro irrigado do Gorutuba. Horticultura Brasileira, Brasília, v. 14, n. 2, p. 220-222, nov. 1996.

SILVA, A. C. F. da; AGOSTINI, I.; MULLER, J. J. V.; VIZZOTO, V. J. Efeito de densidades populacionais sobre a produtividade de pepino para conserva. Horticultura Brasileira, Brasília, v. 10, n. 1, p. 28-29, maio 1992.

SILVA, A. C. F. da; MULLER, J. J. V.; DALL'AGNOL, I.; AGOSTINI, I. Épocas de semeadura e densidade populacional de pepino para conserva. Florianópolis: Empasc, 1988. 16 p. (Comunicado Técnico, 115).

SILVA, A. C. F. da; MULLER, J. J. V.; YOKOYAMA, S. Comportamento de cultivares de pepino para indústria no Baixo Vale do Itajaí, Estado de Santa Catarina. Florianópolis: Empasc, 1979. 7 p. (Comunicado Técnico, 27). 